Role of p53 in Transcriptional Regulation of Proteases in Mammals.

\author{
RAHUL KATARA AND N. SINGH
}

\begin{abstract}
p53, besides being a tumor suppressor protein, is a transcription factor with a powerful activation domain. Mutation(s) in this gene is a frequent event in human carcinogenesis. Several of these mutations lead to loss of its function as transcription factor. Proteases are thought to play an important role in tumor invasion and metastasis by degradation of extra cellular matrices and basement membranes. The function of protein-coding genes is carried out by the protein product, it is important to study p53-dependent expression at the protein level. There are many factors which regulate the expression of proteases in the cell, however here in this review we have focused on the p53 gene and its regulatory effect on the expression of proteases.

\section{INTRODUCTION:}

Proteases are enzymes responsible for degrading proteins. Broadly classified according to their reactive amino acid at the active site, they are either activated as pre-proteins or by disassociation from an inhibitory protein. While they are ubiquitously present in a cell, the distribution of each protease is organelle specific.
\end{abstract}

Department of Biochemistry, All India Institute of Medical Sciences (Rahul Katara), New Delhi ,Department of Biochemistry (N. Singh) J.R...Medical College Gwalior, India.

Correspondence to: RAHUL KATARA

E-mail: sararahi@gmail.com
Lysosomes contain a large variety of hydrolytic enzymes that degrade proteins and other substances taken in by endocytosis. These include many cysteine proteases, as well as some aspartate proteases and one zinc protease. All known lysosomal cysteine proteases are cathepsins, but not all cathepsins are lysosomal or cysteine proteases. ${ }^{1}$ Caspases, calpain and papain are other well known cysteine proteases. Cysteine cathepsins L and B, together with the aspartic protease cathepsin $\mathrm{D}$, are the most abundant lysosomal proteases. Synthesized as inactive precursors, their activation is catalyzed either by other lysosomal enzymes or is autocatalytic in nature and promoted by the acidic $\mathrm{pH}$ within the lysosome.

Gene knockouts have revealed that lysosomal cathepsins have specific and individual functions, which are very important for the normal functioning of an organism. These specific functions are often tissue specific as in cathepsins S and V (immune system) and $\mathrm{K}$ (bone). Although cathepsins B, H, L, F, C, O, $\mathrm{V}, \mathrm{W}$ and $\mathrm{X} / \mathrm{Z}$ are intracellular proteases known to be involved in protein degradation, they are nevertheless involved in some specialized functions like antigen processing, bone remodeling and epidermal homeostasis. ${ }^{2}$ Cathepsin D is a ubiquitous aspartyl, lysosomal endopeptidase. Recent reports suggest its potential as a tumor marker for advanced breast and prostate cancer. Like cathepsin L its normal function is to degrade proteins in the lysosomes. Cathepsins have been shown to be active players in the process of tumorigenesis especially progresion 


\section{Importance of proteases in tumour invasion and metastasis:}

Tumor progression is a step-wise process whereby multiple alterations in normal cells lead initially to a localized tumor, and finally to one that has the ability to invade and metastasize. To be able to migrate from their original site of primary tumor, the tumor cells have to cross several barriers like the basement membranes, interstitial tissues and extracellular matrices. These are composed primarily of collagen, proteoglycans, elastin, laminin and other glycoproteins. This is where the role of these proteases comes in. These proteases catalyze the degradation of interstitial matrix and basement membranes, allowing cancer cells to invade locally and metastasize to distant sites. These include various matrix metalloproteinases (MMPs), urokinase type plasminogen activators (uPA) and lysosomal proteases. ${ }^{2}$ It is thought that these proteases may act in a cascade manner to mediate dissolution of the extracellular matrix.

This is especially evident in mouse models of multistage carcinogenesis where each cathepsin appears to take on a distinct function. ${ }^{3}$ The proteolytic function needs to be finely balanced to promote tumor growth and stage. While angiogenesis and metastasis require localized matrix degradation, a controlled proteolysis is essential to promote cell proliferation and resist apoptosis ${ }^{3}$ Cathepsin D, $\mathrm{B}$ and $\mathrm{L}$ are reported to be play an important role in cancer invasiveness and metastasis. ${ }^{3-6}$

\section{Mechanisms:}

It is apparent then that mechanisms must exist to regulate transcription of the genes encoding each of (the precursors of) these proteinases so that, with the exception of the ubiquitous cathepsin D, expression is facilitated only within certain cells and tissues and is suppressed elsewhere. p53 is one such transcription factor which plays a role in modulating target gene expression. p53 binds to short genomic sequences, called p53-responsive elements, separated by 0 to $13 \mathrm{bp}$, which fits a consensus sequence to variable extent it. p53 is known to regulate proteases such as HTRA2. Jin et al (2003) defined a sequential pathway in mammalian cells in which DNA damage activates p53, which, in turn, increases the transcription of the serine protease gene HTRA2. The interaction of HTRA2 and CIAP1, results in cleavage of CIAP1 and allows caspase to activate apoptosis. ${ }^{7,8}$

The Zinc proteases, matrix metalloprotease (MMP) are involved in degradation of the extracellular matrix during tissue remodeling. Some MMPs have roles in cell signaling relating to their ability to release cytokines or growth factors from the cell surface by cleavage of membrane-bound pre-proteins. For example, MMP-2 is known to mainly degrade type IV collagen and fibronectins and has been implicated in both physiological and pathological conditions, such as trophoblast implantation, wound healing, angiogenesis, arthritis, and tumor cell invasion. A 20-bp perfect p53 binding site was localized within the enhancer region 2 (r2) (a 42-bp oligonucleotide) in the promoter of the MMP-2, and was shown to be regulated by p53 both in vitro and in vivo. ${ }^{9}$ It has also been shown that wt-p53 is able to potently inhibit expression of MMP- $13^{10,11}$ and MMP- $9^{12,13}$. While regulation of proteases by p53 is relatively well studied, p53 mediated regulation of cathepsins is not well elucidated.

It has also been postulated that cathepsin $D$ activates a cascade of proteases involved in the degradation of extracellular matrix by activating cathepsins B and L. ${ }^{14}$ Cathepsin B and $\mathrm{L}$ activity are known to be increased in breast cancer, prostate cancer and early stage gastric carcinomas. Cathepsin K, essentially localized in bone has also been recently shown to promote tumor growth, Interactions of myeloma cells with osteoclasts promote tumour expansion and bone degradation through activation of a complex signaling network and upregulation of cathepsin K, matrix metalloproteinases (MMPs) and urokinase plasminogen activator (uPA). ${ }^{15}$ 
On the other hand, Cathepsin $\mathrm{E}$ has been shown to prevent tumor growth and metastasis. Catalyzing the proteolytic release of soluble TRAIL from tumor cell surface. ${ }^{8}$

It has been reported that mutant $\mathrm{p} 53$ down regulates the expression of protease (cathepsin D) in the case of invasive cancer. ${ }^{16}$ Cathepsin D, the major intracellular aspartyl protease, is a mediator of IFN- $\gamma$ and TNF- $\alpha$ induced apoptosis. There are two p53-specific DNA-binding sites located in the CD promoter responsible for the transcriptional regulation of the cathepsin $\mathrm{D}$ by p53. Previous studies have also demonstrated upregulated expression of Cathepsin D mRNA in some wild type p53 expressing cell lines. ${ }^{17} \mathrm{RT}$ PCR of RNA extracted from tumors from p53 null and p53 wild-type animals confirmed the strongly elevated expression of Cathepsin $\mathrm{E}$ in the p53 null tumors. ${ }^{18}$ Computer assisted data base analysis makes it possible to find the p53 responsive element of all known genes to be putatively trans-activated by p53, some of these genes have been experimentally proven to be trans-activated by p53. ${ }^{19}$ It has been determined that $95 \%$ of these decamers trans-activated by p53 carry cytosine at position 4 and $100 \%$ have guanine at position $7^{20}$

\section{p53 is the key regulator:}

The role of p53 in the biology of cancer is well established and it is one of the most studied tumor suppressor gene in cancer research, ${ }^{21}$ it responds to cell signals by inducing either growth arrest or apoptosis. The activation of apoptosis by p53 is less well understood, but is believed to involve both transcriptional as well as transcription-independent mechanisms. The pattern and extent of induction of different p53 target genes crucially depends on the concentration of active p53 protein within a given cell. ${ }^{18,22}$

\section{Conclusion:}

Based on these reports, it is evident that p53 directly regulates the expression of proteases as in the case of cathepsin D, type IV collagenase and HTRA2 gene. However, the other mechanism(s) underlying this regulation of protease with reference to p53 status and its functional consequence in the cell needs to be further studied.

\section{REFERENCES:}

1 Turk, D. and Guncar, G. Lysosomal cysteine proteases (cathepsins): promising drug targets. Acta Crystallogr D Biol Crystallogr. 2003;59:203-213.

2 Turk V, Turk B, Guncar G, Turk D, Kos J. Lyso'somal cathepsins: Sturcture, role in antigen processing and presentation and cancer. Adv Enzyme Regul: 2002;42:285-303

3 Joyce JA, Baruch A, Chehade $K$, et al. Cathepsin cysteine proteases are effectors of invasive growth and angiogenesis during multistage tumorigenesis. Cancer Cell 2004;5:443-453.

4 Kos J, Lah TT. Cysteine proteinases and their endogenous inhibitors : target proteins for prog nosis, diagnosis and therapy in cancer (review). Oncol Rep 1998;5:1349-1346.

5 Foekens JA, Look MP, Bolt-de Vries J, et. al. Cathepsin $D$ in primary breast cancer : prognostic evaluation involving 2810 patients. Br J Cancer 1999;79:300-307.

6 Lah TT, Cercek M, Blejec A, et. al. Cathepsin B, a prognostic indicator in lymph node-negative breast carcinoma patients : comparison with cathepsin $D$, cathepsin $L$, and other clinical indicators. Clin Cancer Res 2000;6:578-584.

7 Shengkan Jin, Markus Kalkum, Michael Overholtzer, et. al. CIAP1 and the serine protease HTRA2 are involved in a novel p53-dependent apoptosis pathway in mammals. Genes Dev. 2003;17:359-367.

8 Kawakubo T, Okamoto K, Iwata J, et al. Cathepsin E Prevents Tumor Growth and Metastasis by Catalyzing the Proteolytic Release of Soluble TRAIL from Tumor Cell Surface. Cancer Res. 2007;67(22):10869-78.

9 Junhui Bian and Sun Yi. Transcriptional Activation by p53 of the Human Type IV Collagenase (Gelatinase $A$ or Matrix Metalloproteinase 2) Promoter. $M C B$. 1997;17:6330-6338.

10 Sun $Y$, Cheung JM, Martel-Pelletier $J$, et. al. Wild type and mutant p53 differentially regulate the gene expression of human collagenase-3 (hMMP-13). J Biol Chem. 2000;14:275(15):11327-32

11 Sun $Y$, Zeng XR, Wenger L, Firestein GS, Cheung HS, P53 down-regulates matrix metalloproteinase-1 by targeting the communications between AP-1 and the basal transcription complex. J Cell Biochem 2004; 15:258-69.

12 Liu J, Zhan M, Hannay JAF. Wild-type p53 inhibits nuclear factor-B-induced matrix metalloproteinase- 9 promotor activation: Implications of soft tissue sarcoma growth and metastasis. Mol Cancer Res) 2006;4:803-810. 
Meyer E, Vollmer JY, Bovey R, Stamenkovic I. Matrix metalloproteinases 9 and 10 inhibit protein kinase $C$ potentiated, p53-mediated apoptosis. Cancer Res 2005;65:4261-4272.

14 Turk V, Turk B, Guncar G, Turk D, Kos J. L.yso'somal cathepsins: Sturcture, role in antigen processing and presentation and cancer. Adv Enzyme Regul: 2002;42:285-303.

15 Hecht $M$, von Metzler I, Sack K. Interactions of myeloma cells with osteoclasts promote tumour expansion and bone degradation through activation of a complex signalling network and upregulation of cathepsin K, matrix metalloproteinases (MMPs) and urokinase plasminogen activator (uPA) Exp. Cell Res. 2008;314(5):1082-1093.

16 Losch V, Schindl M. Kohlberger P. et. al. Cathepsin D in ovarian cancer: prognostic value and correlation with p53 expression and microvessel density. Gynecol Oncol 2004;92:545-552.

Wu GS, Saftig P, Peters $C$ and El-Diery WS Potential role for Cathepsin D in p53-dependent tumor suppression and chemosensitivity. Oncogene, 1998;16:2177-83.
18

19

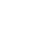

Lewis BC Klimstra DS, Socci ND et. al. The Absence of p53 Promotes Metastasis in a Novel Somatic Mouse Model for Hepatocellular Carcinoma Mol. Cell. Biol.2005;25:1228-1237

9 Inga, A., Storici, F., Darden, T. A. \& Resnick, M. A. Differential Transactivation by the p53 Transcription Factor Is Highly Dependent on p53 Level and Promoter Target Sequence Mol. Cell. Biol.2002;22:8612-25.

20 Bourdon J-C Deguin-Chambon V, Lelong JC et. al. Further characterization of the p53 responsive element-identification of new candidate genes for trans-activation by p53. Oncogene 1997;14:85-94.

Levine, A. J. p53, the cellular gatekeeper for growth and division Cell 1997;88:323-331.

Lo, P. K., Chen, J. Y., Tang, P. P. et. al. Identification of a Mouse Thiamine Transporter Gene as a Direct Transcriptional Target for $p 53^{*} \mathrm{~J}$. Biol. Chem. 2001;276:37186-93.

\title{
Change of Address
}

Dr sudeep Gupta will be Editor for the Indian Journal of Medical \& Paediatric Oncology from January, 2009 onwards. Kindly send your manuscripts on following address:

\author{
Dr Sudeep Gupta
}

Associate Professor of Medical Oncology

Editor, Indian Journal of Medical \& Paediatric Oncology

Tata Memorial Hospital

Parel Mumbai 400012

E-Mail : sudeepgupta04@yahoo.com

sudeepgupta@tatahospital.net 